\title{
PROGRAMA PAPÁ DESDE ADENTRO: ESTRATEGIAS Y HABILIDADES PARENTALES PARA PADRES PRIVADOS DE LA LIBERTAD
}

\section{PROGRAM "PAPÁ DESDE ADENTRO": PARENTING STRATEGIES AND SKILLS FOR PARENTS DEPRIVED OF THEIR LIBERTY}

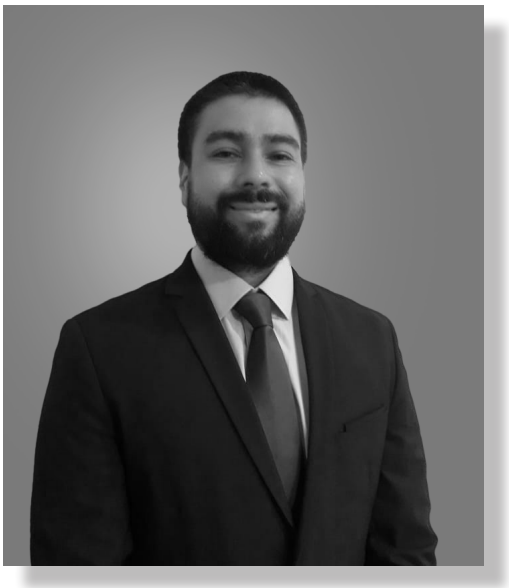

Sebastián Ignacio Fernández Díaz

Maestro en Desarrollo Humano por la Universidad

Iberoamericana, Ciudad de México.

fernandez.soc@.gmail.com

Chile

\section{RESUMEN}

El presente artículo sintetiza y rescata una serie de aprendizajes y experiencias de un programa de intervención para el desarrollo de estrategias parentales para padres privados de la libertad de la ciudad de México, que se realizó entre el año 2018 y el 2020. Este trabajo comenzó con una etapa de diagnóstico que incluyó a privados y privadas de libertad (primo-delincuentes y reincidentes), familiares de los internos e internas, directores/as de los centros penitenciarios y ex internos e internas (no reincidentes). Con la información recopilada y analizada se adecuaron estrategias del programa Triple $\mathrm{P}$ y se revisó literatura proveniente del enfoque centrado en la persona, del enfoque en parentalidad positiva y sobre género y nuevas masculinidades, para adecuar un programa que contempló la realidad de los padres privados de libertad del Centro de Ejecución de Sanciones Penales Varonil Norte. A un mes de finalizado el taller se aplicó una segunda evaluación para poder medir su impacto.

PALABRAS CLAVES: Padres privados de libertad, Parentalidad Positiva, Triple $\mathrm{P}^{1}$, Desarrollo Humano, Ciudad de México.

\begin{abstract}
This article synthesizes and rescues a series of learnings and experiences from an intervention program for the development of parental strategies for parents deprived of their liberty in Mexico City, which took place between 2018 and 2020. This work began with a diagnostic stage that included deprived of liberty (first offenders and recidivists), relatives of the inmates, prison directors and former inmates (non recidivists). With the information gathered and analyzed, strategies of the Triple $\mathrm{P}$ program were adapted and literature was reviewed that came from the focus on the person, the focus on positive parenting and on gender and new masculinities, in order to adapt a program that contemplated the reality of the parents deprived of liberty in the North Men's Penalty Execution Center. One month after the end of the workshop a second evaluation was applied in order to measure its impact.
\end{abstract}

WORDS: Parents deprived of liberty, Positive Parentality, Triple P, Human Development, Mexico City.

\section{INTRODUCCIÓN}

A principios del año 2018 como parte del trabajo realizado en la Fundación Amaneceres en la Obscuridad $^{2}$, se creó un programa en "habilidades parentales" para padres privados de la libertad de la Ciudad de México. La iniciativa comenzó a propósito del diagnóstico y evaluación sobre la oferta programática de la fundación. Este diagnóstico incluyó a privados y privadas de libertad primodelincuentes (que cumplían una sentencia por primera vez) y reincidentes (que están cumpliendo sentencia

1 Triple P, Programa de Parentalidad Positiva: es un sistema de apoyo a los padres, madres y familias, diseñado para prevenir y tratar - los problemas conductuales y emocionales en niños, ninas y adolescentes. Este programa es basado en la evidencia y es de origen australiano.

2. Fundación dediçada por más de 17 años al acompañamiento, educación, capacitación, reinserción dignificación de las condiciones de los/as privadas/os de libertad de la Ciudad de México. 
por segunda o más veces), familiares de los internos e internas, directores/as de los centros penitenciarios $\mathrm{y}$ ex internos e internas (no reincidentes).

Entre los resultados más relevantes extraídos de los análisis de datos de las entrevistas en profundidad y los grupos focales se encontró que en el grupo de hombres privados de libertad existía una gran preocupación y una necesidad por mantener, recuperar y restaurar el vínculo con sus hijos e hijas, pero que lamentablemente carecían de las herramientas, la confianza y muchos de ellos concebían como truncada o anulada la posibilidad de seguir siendo padres una vez que se encontraban cumpliendo una pena con privación de libertad. Al mismo tiempo, hijos e hijas de ex internos manifestaron que este vínculo había sido significativo para su desarrollo en la niñez y adolescencia a pesar de la privación de libertad de sus padres. A su vez, los privados de libertad mencionaron que el vínculo con sus hijos e hijas era una experiencia que les daba sentido a su vida, además de ser un aliciente para iniciar un proceso de desistimiento delictual.

Con la información extraída del diagnóstico, se adecuaron estrategias y se revisó la literatura proveniente del enfoque centrado en la persona, del enfoque en parentalidad positiva, del programa Triple P y sobre teoría de géneros y nuevas masculinidades, para adecuar de forma vivencial un proyecto-taller que contempló la realidad de los padres privados de libertad del Centro de Ejecución de Sanciones Penales Varonil Norte, ubicado en la zona norte de la Ciudad de México. Al mes de terminado el taller se volvió a aplicar un instrumento de evaluación para poder medir el impacto del taller.

\section{MARCO CONTEXTUAL}

El trabajo se realizó en dos centros penitenciarios, se comenzó el diagnóstico general en la penitenciaría del Distrito Federal de la ciudad de México, allí se entrevistó a privados de libertad, a ex privados de libertad de ese centro penitenciarios (no reincidentes), a la directora del centro y a familiares de ex privados de libertad.

Una vez concluido el diagnóstico y analizados los datos se presentó la propuesta de un programa que desarrollaba habilidades y estrategias parentales a la Subsecretaría de Penitenciarias de la Ciudad de México, en el marco de la oferta programática de la Asociación Amaneceres en la Obscuridad.
Por recomendación y solicitud de la Subsecretaría antes mencionada, el programa se implementó en el Centro de Ejecución de Sanciones Penales Varonil Norte, debido a que las características de este último lo hacían idóneas para la implementación del programa. Ya que allí se concentraban padres privados de la libertad cumpliendo sanciones más cortas (menos de dos años, debido a su buen comportamiento en el cumplimiento de sus penas totales, es un tipo de cárcel modelo) (Subsecretaria de Sistema Penitenciario CDMX, 2020) en comparación a la penitencia del Distrito Federal en donde se encuentran privados de libertad cumpliendo penas particularmente altas, treinta (30), cuarenta (40) años o más en la mayoría en muchos casos. La segunda parte del diagnóstico, la implementación y evaluación se realizaron en el segundo centro penitenciario (Centro de Ejecución de Sanciones Penales Varonil Norte).

\section{METODOLOGÍA}

Este trabajo fue de tipo cualitativo, un estudio de caso intrínseco, ya que lo que se buscó fue conocer y comprender la realidad de los internos para contar con herramientas para poder generar un programa que se ajuste a su realidad. El estudio de caso intrínseco busca estudiar el caso en sí mismo, y el instrumental se utiliza para someter a prueba una teoría (Muñiz, 2010). Para efectos de esta investigación, el tipo de estudio de caso que más se adecua según los objetivos y la población estudiada es el estudio de caso, de caso atípico (Muñiz, 2010). Debido a que la población con la que se trabaja posee una característica atípica, su condición de internos del sistema penitenciario, más allá de su condición de ser padre, pareja o de querer mejorar la relación con sus hijos e hijas.

Como técnicas de recolección de datos se aplicaron entrevistas en profundidad semiestructuradas (Cea, 2001) a privados de libertad, en calidad de primodelincuentes y reincidentes, además se entrevistó a la directora del centro penitenciario. También para complementar el diagnóstico se realizaron grupos focales a ex internos y a familiares y amigos de estos. Esta entrevista tiene por objetivo que los entrevistados hablen de sus experiencias familiares, de su relación con sus padres, de su propia experiencia y de su significación de ser padre e interno. Por otro lado, también se buscó con esta entrevista ahondar en sus percepciones, temores, expectativas y problemáticas con respecto a la salida del penal. 
La segunda técnica es el focus group, definida esta como una reunión de un grupo de individuos seleccionados por los investigadores para discutir o elaborar desde la experiencia personal, una temática o hecho social que es objeto de investigación (Chitarroni, 2008). Con esta técnica se realizaron dos grupos focales el primero se realizó con internos que representan experiencias exitosas de reinserción (ex internos no reincidentes) y el segundo se realizó con los familiares en el exterior de la cárcel. Esto con el objetivo de comprender los temores y expectativas del grupo al momento de salir del penal, las dificultades y problemáticas a las que se enfrentaron y cómo las resolvieron. También se indagó en sus experiencias familiares, el significado que le atribuyen a ser papá y en las dificultades que implicaron el regreso a casa después del periodo de sentencia.

Con respecto al tipo de muestreo, en este caso fueron utilizados dos. El primero es de tipo ideal, definido como muestreos de tipo no probabilísticos, que se utilizan principalmente en estudios cualitativos, en donde el objetivo principal es la riqueza, profundidad y calidad de la información. No la cantidad ni la estandarización (Hernández, et al., 2010).

El segundo tipo de muestreo es de intencionalidad o de conveniencia. En este se escogen las unidades a entrevistar siguiendo criterios de conveniencia del investigador o de los objetivos de la investigación (riqueza de información en el caso, posición que ocupa en relación al fenómeno estudiado, etc.). En este caso, encontramos un informador clave, y este identificó a otro a quien entrevistar y así sucesivamente. De igual manera, el investigador decide qué individuos de la población pasan a formar parte de la muestra en función de la disponibilidad de los mismos, proximidad con el investigador o por factibilidad del estudio (Departamento Sociología Universidad Alicante, s.f.).

Con respecto a la triangulación de datos, definida como la utilización de diferentes fuentes y métodos de recolección (Hernández, et al., 2010) como se mencionó anteriormente existen cinco fuentes de información y dos métodos de recolección. Entrevistas en profundidad a primo-delincuentes (delincuentes primarios), entrevistas en profundidad a reincidentes, a la directora del penal, grupo focal a ex internos y grupo focal a sus familiares, para darle una mayor riqueza y tener una mayor amplitud de cómo viven la parentalidad los internos, su proceso de sentencia y su egreso de los centros penitenciarios. Con respecto a la recolección y análisis de la información, debido a la disposición del penal se realizó un registro de audio de las entrevistas realizadas a los internos por medio de una grabadora de voz, pero no fue posible realizar tomas de fotografías ni grabaciones en video, de modo que el análisis de los datos se apoya principalmente en un registro de audio que luego fue transcrito y posteriormente categorizado o codificado.

Este último se pude definir como:

El modo en que usted define de qué tratan los datos que está analizando. Implica identificar y registrar uno o más pasajes de texto u otros datos como parte de cuadros que, en cierto sentido, ejemplifican la misma idea teórica o descriptiva. (Gibbs, 2007, p.64) Esta codificación, procesamiento $\mathrm{y}$ análisis se realizó a través del software para análisis de datos cualitativos QDA ${ }^{3}$ Miner.

\section{FASES DEL TRABAJO}

El trabajo estuvo dividido en cuatro etapas o fases: Una fase de diagnóstico, una fase de adaptación, una etapa de implementación y una de evaluación.

\section{Fase de diagnóstico}

Esta fase a su vez, tuvo dos etapas, la primera de ellas se realizó a internos, ex internos, familiares de ex internos y directora de la Penitenciaría Del Distrito Federal (ver Tabla 1) y la segunda a privados de la libertad del Centro de Ejecución de Sanciones Penales Varonil Norte. En la primera etapa se realizaron entrevistas en profundidad a privados de libertad primo delincuentes y reincidentes, entendiendo los primeros como privados de libertad cumpliendo una sentencia por primera vez y los segundos como privados de libertad que se encuentran cumpliendo una sentencia por segunda vez o más por el mismo delito o uno similar (Organización de las Naciones Unidas [ONU], 2013). También se entrevistó a la directora del centro penitenciario.En total en esta etapa de diagnóstico se entrevistó a diez (10) internos primo-delincuentes, tres (3) reincidentes, una (1) directora de centro penitenciario, cinco (5) ex internos y cinco (5) familiares de ex internos. Un total de veinticuatro personas (24) participaron en La segunda etapa del diagnóstico se realizó en Centro de Ejecución de Sanciones Penales Varonil Norte, 


\section{TABLA 1}

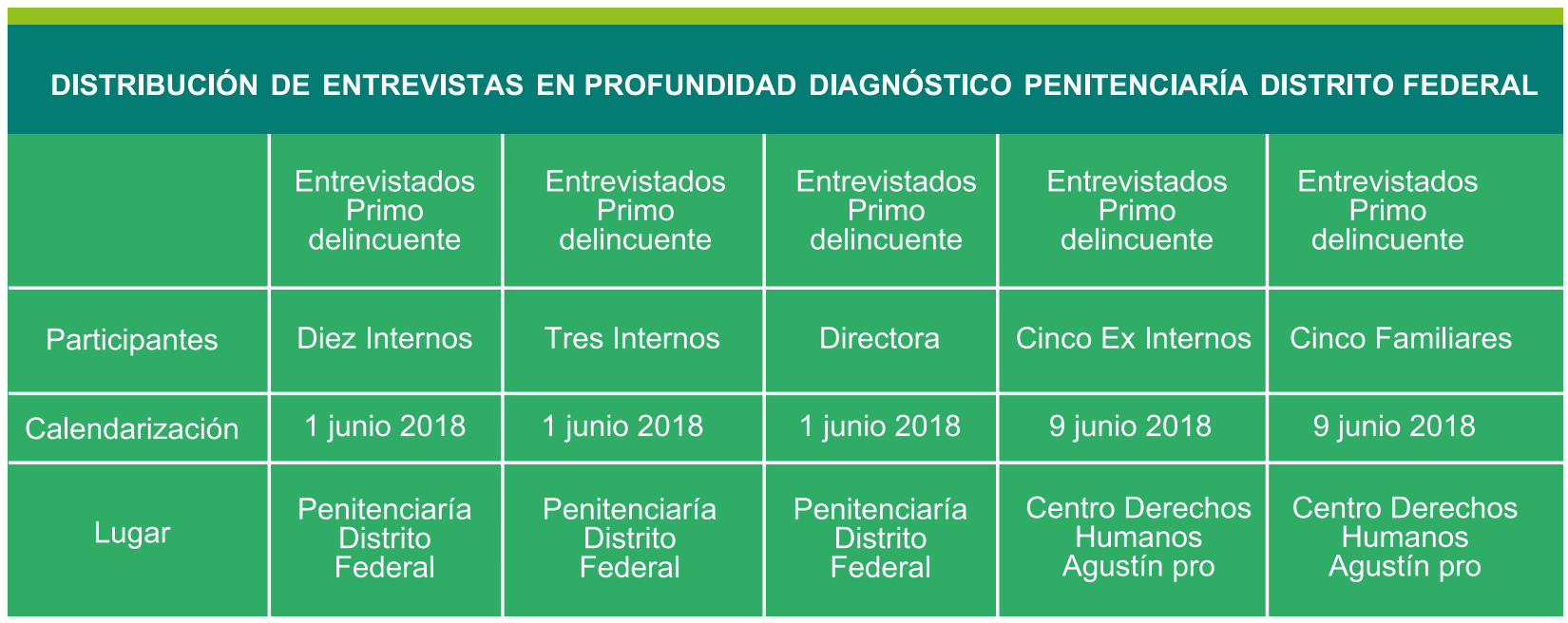

a recomendación y solicitud de la Subsecretaria de Penitenciarías. Se aplicó el Instrumento de evaluación de habilidades parentales para padres privados de libertad a los siete (7) participantes con los que se inició (a pesar de en el transcurso del taller se agregaron más, no se consideraron para el análisis final de la evaluación). El instrumento contó con 14 preguntas abiertas en donde se explora su concepto de parentalidad, sus estrategias parentales, el conocimiento del impacto de su privación de libertad en sus familias y las certezas sobre su salida del centro penitenciario.

\section{Fase de Adaptación}

Se tomó como referencia para la creación del proyecto el seminario, El poder de la parentalidad positiva, del programa Triple P (Triple P, 2009) y se adaptó a las necesidades y el contexto de los internos en base a la información extraída de los diagnósticos de ambos centros penitenciarios. 


\section{TABLA 2}

\begin{tabular}{|c|c|c|c|c|}
\hline \multicolumn{2}{|c|}{ SESIÓN ORIGINAL } & \multicolumn{3}{|c|}{ INTERVENCIÓN DISEÑADA } \\
\hline Sesiones & Original & Sesión & $\begin{array}{l}\text { Propuesta de } \\
\text { intervención internos }\end{array}$ & Sesiones \\
\hline \multirow[t]{5}{*}{$\begin{array}{l}\text { Sesión } \\
\text { Dos horas }\end{array}$} & $\begin{array}{l}\text { Proporcionar } \\
\text { un ambiente } \\
\text { seguro e } \\
\text { interesante }\end{array}$ & $\begin{array}{l}\text { Sesión } 1 \\
\text { Dos horas }\end{array}$ & Ser padre interno & $\begin{array}{l}\text { Se agrega a la propuesta original } \\
\text { debido a los resultados del } \\
\text { diagnóstico en relación a la falta de } \\
\text { significación de lo que es ser un } \\
\text { padre interno. }\end{array}$ \\
\hline & $\begin{array}{c}\text { Proporcionar un } \\
\text { ambiente } \\
\text { positivo }\end{array}$ & $\begin{array}{l}\text { Sesión } 2 \\
\text { Dos horas }\end{array}$ & $\begin{array}{l}\text { Estrategias } \\
\text { parentales } 1\end{array}$ & $\begin{array}{l}\text { Incluye los puntos } 1,2 \text { y } 3 \text { de la } \\
\text { sesión original del seminario Triple } \\
\mathrm{P} \text {, en base a la carencia de } \\
\text { estrategias parentales de los } \\
\text { internos. }\end{array}$ \\
\hline & $\begin{array}{c}\text { Utilizar disciplina } \\
\text { asertiva }\end{array}$ & $\begin{array}{l}\text { Sesión } 3 \\
\text { Dos horas }\end{array}$ & $\begin{array}{c}\text { Estrategias } \\
\text { parentales } 2\end{array}$ & $\begin{array}{l}\text { Incluye los puntos } 4 \text { y } 5 \text { de la sesión } \\
\text { original del seminario Triple } \mathrm{P} \text {, en } \\
\text { base a la carencia de estrategias } \\
\text { parentales de los internos. }\end{array}$ \\
\hline & $\begin{array}{l}\text { Tener expectati- } \\
\text { vas realistas }\end{array}$ & $\begin{array}{c}\text { Sesión } 4 \\
\text { Dos horas }\end{array}$ & $\begin{array}{l}\text { El impacto en } \\
\text { la familia }\end{array}$ & $\begin{array}{l}\text { Se agrega a la propuesta original } \\
\text { debido a los resultados del } \\
\text { diagnóstico en donde se } \\
\text { desconocen los impactos del } \\
\text { internamiento en la familia. }\end{array}$ \\
\hline & $\begin{array}{l}\text { Cuidarse a sí } \\
\text { mismos como } \\
\text { padre o madre }\end{array}$ & $\begin{array}{l}\text { Sesión } 5 \\
\text { Dos horas }\end{array}$ & $\begin{array}{l}\text { Construyendo } \\
\text { certezas }\end{array}$ & $\begin{array}{l}\text { Se agrega a la propuesta original } \\
\text { debido a los resultados del } \\
\text { diagnóstico, en donde los internos } \\
\text { carecen de información relacionada } \\
\text { con su salida del centro } \\
\text { penitenciario. }\end{array}$ \\
\hline
\end{tabular}

El seminario contemplaba 1 sesión de dos horas aproximadamente (Turner, et al., 2013) y finalmente la intervención diseñada quedó definida con un total de 5 sesiones de dos horas cada una (ver tabla 2), para lograr abarcar las problemáticas más importantes mencionadas por los padres privados de libertad. Estas incluyeron además de las estrategias parentales; su concepción de que es ser padre, el ejercicio de esta (como serlo) y el impacto del internamiento en su forma de ser padre. También se exploró el impacto del internamiento en sus familias y sus temores y expectativas con respecto a su salida del centro penitenciario. Esto con la finalidad de que fueran construyendo algunas mínimas certezas para su egreso del centro penitenciario y para retomar la convivencia diaria con sus familias, la sociedad y sus hijos e hijas. 


\section{Fase de implementación}

Esta fase fue posible ya que este trabajo se enmarcó como parte de un proyecto piloto a propósito de la evaluación anual de la Asociación Amaneceres en la Obscuridad. Una vez que se obtuvo la aprobación del proyecto piloto por parte de la Subsecretaria de Penitenciarias de la Ciudad de México, se procedió a generar y enviar los oficios para la aprobación de los recursos físicos y tecnológicos para la ejecución del proyecto. Los recursos que se solicitó autorización de ingresar fueron: una laptop, un proyector, lápices, hojas, galletas (para meriendas de los participantes) y libretas para las anotaciones de los asistentes. El proceso desde que se presentó el proyecto hasta su autorización fue de aproximadamente tres meses. Una vez autorizado el proyecto y el ingreso, hubo un primer acercamiento con el Centro de Ejecución de Sanciones Penales, allí se contactó con el personal encargado y se definieron fechas para la ejecución de las sesiones, el lugar y sobre cómo se procedería.

\section{Fase de evaluación}

Se realizó una primera evaluación o diagnóstico (ex ante) de los padres privados de libertad, que participarían del taller, se les aplicó el Instrumento de evaluación de habilidades parentales para padres privados de libertad. El instrumento contó con catorce (14) preguntas abiertas en donde se exploró: su concepto de parentalidad, sus estrategias parentales, el conocimiento del impacto de su privación de libertad en sus familias y las certezas sobre su salida del centro penitenciario. Este instrumento de evaluación ex ante o diagnóstico fue aplicado a siete (7) padres privados de libertad.

Para evaluar el impacto de la intervención (ex post), se aplicó a un mes de la intervención el mismo instrumento para medir si a un nivel cognoscitivo aportó a la definición de su concepto de parentalidad, a conocer una serie de estrategias parentales y conocimiento del impacto de su privación de libertad en sus familias. También se recopilo información sobre su conocimiento acerca de estrategias que le ayudarán a mejorar la relación con sus familias (sobre todo hijos e hijas) y sobre la construcción de certezas que apoyen su proceso de salida del centro penitenciario y posterior reintegración a distinto espacios (familiares, laborales, barrio, etc.). Esta evaluación ex post fue aplicada a los mismos siete (7) padres privados de libertad a los que cuales se les aplicó el instrumento ex ante inicialmente. Se sumaron a esta evaluación cinco (5) padres privados de libertad más, estos se fueron integrando en el transcurso de las sesiones, pero no se consideraron en el análisis ya que no participaron de la evaluación ex ante o diagnóstica.

Es decir, el instrumento final (ex post) se aplicó a un total de doce (12) padres privados de libertad, pero para el análisis se consideraron siete (7).

\section{ANÁLISIS DE LOS RESULTADOS}

Para efectos prácticos y para presentar los resultados obtenidos y su análisis, se realizaron apartados por cada una de las categorías o ámbitos revisados. En cada una de ellas se presentarán los datos extraídos del diagnóstico o evaluación inicial, citas de las evaluaciones y una pequeña síntesis del análisis de los datos.

\section{Concepto de parentalidad y consecuencias del internamiento en su parentalidad}

Se indagó en los internos sobre qué significaba para ellos ser padres y privados de liberad, el grupo mayor de ellos menciona la dificultad de la tarea de ser padres cumpliendo una sentencia y la definen como una tarea incompleta. Mencionan sentirse frustrados e imposibilitados de ser padres, a pesar de querer realizar la labor. También describen su rol de padre en tareas limitadas y específicas como sólo dar educación, dar cariño o apoyar económicamente. En general se sienten incompletos e irresponsables como padres ya que se conciben como limitados e imposibilitados para continuar con la labor de padre según su significación y debido a la falta de presencia física. Solo un par de ellos mencionan un rol más integral en la crianza de los hijos y establecen que desde su internamiento también siguen criando y siendo padres.

Es algo difícil en la vida para mí el no poder con mis hijos. No puedo estar presente ni en su educación ni en sus logros. (L. Castro, entrevista Centro de Ejecución de Sanciones Penales Varonil Norte, 14 de enero 2019).

Después de la intervención y en relación a esta categoría se puede observar que los entrevistados mencionaron discursos más responsables y esperanzadores con respecto a la primera evaluación, en donde su condición de internamiento limitaba y casi anulaba su rol de padre. En general los padres 
mencionaron después de la intervención que, si bien es una situación difícil, pueden continuar con su labor de padres desde dentro del centro penitenciario y que no será para siempre. También se puede rescatar el reconocimiento de la valía o valoración de su presencia o existencia en la vida de su hijos/as y su familia y la necesidad de mantener este vínculo ligado a los beneficios emocionales recíprocos.

Es la posibilidad de relacionarme bien con ellos en todas sus etapas. Niño, adolescente y adulto. Y poder crear sólidos lazos afectivos en ellos y tener una agradable crianza. Es tener una responsabilidad con la familia, estar ausente, pero seguir desde aquí con ella. (D. Leiva, entrevista Centro de Ejecución de Sanciones Penales Varonil Norte, 21 febrero 2019).

Impacto en la familia en el proceso de encarcelamiento Después de la intervención y frente a esta categoría, se observa en el discurso de los internos una mayor conciencia sobre las consecuencias del internamiento en sus familias, entre ellas mencionan la agresión de amigos y amistades a los hijos/as, el alejamiento de la familia más extensa a su familia nuclear (pareja, hijos/as), el hecho de que su familia se sienta sola, el descuido de su familia en diversos ámbitos; entre ellos lo económico, lo moral, lo escolar, además de percatarse de que les afecta su propia ausencia y aportes.

Son muchas las consecuencias, algunas son irreversibles. Pero hoy sé que descuidé a mis hijos en lo económico, en lo moral, en lo escolar, etc. (D. González, entrevista Centro de Ejecución de Sanciones Penales Varonil Norte, 21 de febrero 2019).

Después de la intervención y frente a esta categoría, se observa una diferencia en el discurso de los internos en relación a la primera evaluación, se puede identificar un mayor conocimiento o conciencia de la diversidad de consecuencias que tuvo su internamiento, a pesar de mencionar solo algunas de esas consecuencias.

\section{Expectativas y temores al terminar su proceso de sentencia}

Después del taller y de ser analizadas sus respuestas se observa una mayor profundidad y desarrollo en cuanto a la descripción de sus expectativas y temores con respecto a su salida del penal. Las expectativas se convierten en temores cuando se presenta su imposibilidad; entre estos últimos mencionan la discriminación familiar, social, laboral y el desempleo. Al preguntarles sobre si tenían algún plan para resolverlos, muy pocos de ellos mencionaron un plan concreto para resolverlos, más bien nombraron disposiciones que les permitirían poder acercarse a ellos, pero no acciones concretas.

Mis mayores temores son la falta de empleo y la discriminación por haber estado aquí... Entre mis planes para lograrlo se encuentran, hablar con la verdad y sobre todo con amor a mi familia. S. Romero. (entrevista Centro de Ejecución de Sanciones Penales Varonil Norte, 21 de febrero 2019).

$\mathrm{Al}$ analizar esta categoría, al terminar su proceso de sentencia, se puede desprender que, si bien hubo un cambio en su discurso en donde los internos logran darle una mayor profundidad las problemáticas relacionadas con sus expectativas y temores, la mayoría de ellos al momento de preguntarle sobre un plan de acción para resolverlos, no mencionaron un plan concreto, excepto dos de ellos. Los demás mencionan actitudes para resolver estas problemáticas, para que los reciban mejor y para poder adaptarse, pero no mencionan un plan. Desde esta perspectiva se propone revisar esta parte del taller para poder determinar cómo se puede mejorar.

\section{Estrategias Triple $\mathbf{P}$ y estrategias utilizadas por los internos}

Después de la intervención, los entrevistados mencionaron que además de las llamadas telefónicas, utilizaban como una estrategia el conversar y escuchar a sus hijos e hijas con frecuencia, el estar permanentemente comunicados, el apoyo constante y la presencia permanente; además del afecto y la cercanía para estar presentes en la vida de sus hijos e hijas.

Con respecto a la disciplina mencionaron que platicaban con ellos para ayudarles a establecer su propio criterio, que le ponen límites a su conducta y se apoyan en la cuidadora de sus hijos/as para estas labores. También buscaban instancias para hablarles de valores y principios, sobre las consecuencias de los actos. Solo uno de ellos mencionó no poder hacerlo, argumentando que "no tenía cara para 
decirlo", haciendo referencia a la incongruencia de ser un referente moral para sus hijos si se encontraba cumpliendo una sentencia.

Con respecto a las estrategias para enseñar mencionaron utilizar principalmente el ejemplo (modelaje), el diálogo, la comprensión y se esforzaban por ser amistosos con sus hijos/as. En cuanto a las formas de demostrar afecto mencionan; abrazos, caricias, diciéndoles que los aman, tratando de demostrárselo de distintas formas, en cada momento que pueden, solo mencionan formas cercanas $\mathrm{y}$ amables.

En cuanto a las necesidades y cuidados de los niños/as mencionan la diversidad de ellos; amor, respeto, educación, cuidado, seguridad, escucha y la presencia de ellos mismos como padres. Algunos de ellos establecieron ser conscientes de que no sabían las necesidades de sus hijos/as, pero que se estaban esforzando por aprenderlas.

Al preguntarles sobre expectativas en sus hijos e hijas declaran que esperan de ellos y ellas que sean buenas personas, que puedan realizarse y desempeñarse en lo que ellos/as quieran, que logren ser felices y alcanzar sus metas; una gran parte menciono el que no cometan los mismos errores que ellos.

Haciéndoles saber las consecuencias y pues no prohibiéndoles si no haciéndoles saber lo que pude pasar. Con buenas palabras con cariño y respeto... Para que sea una persona de bien y no sigan los malos ejemplos que hice antes. (D. Ramírez, entrevista Centro de Ejecución de Sanciones Penales Varonil Norte, 21 de febrero 2019).

Con respecto a sus expectativas como padres mencionaron: poder apoyarlos, acompañarlos y poder ser un buen ejemplo para ellos, además de poder darles una buena educación.

En cuanto a las estrategias de autocuidado, aquí es donde se observa el gran déficit, ya que la gran mayoría de ellos mencionaron no realizarlas. Solo tres de los internos describieron ir a terapias, hacer ejercicios y cuidar de sí mismos.

En esta categoría se puede concluir que después de la intervención, hubo un cambio en el discurso de los internos. Se puede observar un mayor reconocimiento y descripción de actividades en las que ellos pueden estar presentes en la vida de sus hijos e hijas.
Se identifica también una presencia generalizada en su discurso: del afecto, la confianza y el apoyo como estrategias constantes y básicas en la crianza de sus hijos e hijas. Se observa en su discurso una mayor conciencia del desconocimiento con respecto a las necesidades de sus hijos e hijas, pero que es algo que se puede subsanar. Se pudo observar expectativas realistas con respecto a sus hijos e hijas, que descansan en las que se plantee el niño o niña y expectativas realistas de ellos como padres en su condición de privado de libertad. Desde esta condición se percibe la autorregulación y la voluntad de que ellos puedan ser un buen ejemplo a través del modelaje. No se logró observar un cambio significativo a nivel discursivo y de manera generalizada, en el ámbito de las estrategias de autocuidado de los padres, (estudios en nuevas masculinidades mencionan que este es un aspecto generalizado en los hombres) solo un par de ellos mencionaron las terapias, su propio autocuidado y el ejercicio físico como estrategias de cuidado.

\section{DISCUSIÓN}

El trabajo de investigación e intervención en el sistema penitenciario en México, presenta una serie de problemáticas que dificultan el trabajo en él. Entre las más complejas se encuentra los excesivos trámites burocráticos y los vacíos de tiempo que quedan entre la entrega de un documento y la autorización de este. En el caso de esta intervención pasaron tres meses entre la entrega y la autorización del ingreso y el programa. Por otro lado, existe una falta de coordinación entre la Subsecretaria de Penitenciarías y quienes difunden y gestionan los espacios y la información dentro de los centros penitenciarios. Es decir, lo que dice la organización central se hace, sin considerar los recursos y actividades de los internos. Estos factores dificultaron la obtención de un espacio físico, que hubo que gestionar al momento del ingreso, también al momento del ingreso. Hubo que adaptar una serie de procedimientos ya que el horario del taller coincidía en paralelo con otros cursos obligatorios de los internos. También se supo que la información sobre el taller había llegado el día anterior y la encargada de difusión no había podido informar sobre el taller a los internos, por lo que hubo que realizar una difusión del taller boca a boca, ese mismo día, por el patio del centro penitenciario. Además, se dio a conocer el taller como una escuela 
para padres, sin dar la información detallada sobre los objetivos y contenidos.

Una vez que se llevó a cabo la primera sesión y al darse cuenta algunos internos que no era solo una escuela para padres, comenzaron a comentar entre ellos sobre las sesiones y los aprendizajes; luego de esto el número de participantes pasó de 7 a 12 internos, fue aumentando a medida seguían las sesiones. Aun así y siguiendo la obligatoriedad de los talleres a los que ya estaban inscritos algunos internos, se ausentaban en parte de las sesiones y volvían, se generaba una suerte de rotación. La mayoría de ellos asistieron a casi todo el taller y no se ausentaron debido a que estaban prontos a salir (menos de un mes), por lo que ya no debían llevar los cursos obligatorios y estaban interesados en los contenidos del taller y les daba confianza para enfrentar su pronta salida e inminente encuentro con sus familias, hijos/hijas y el exterior mismo. De hecho, algunos de ellos no se les pudo aplicar el instrumento de evaluación ex post, debido a que su liberación sucedió antes de esta aplicación a un mes de la intervención, esta situación es una limitante para poder evaluar ya que los internos no saben con certeza el día de su liberación.

\section{CONCLUSIONES}

Si bien, la mayoría de hombres privados de libertad en México son padres y una gran cantidad de ellos se ven interesados en capacitaciones de estas características, no todos pueden acceder a estos programas debido a distintos factores, entre estos que muchos de ellos presentan antecedentes que pueden representar un peligro para el bienestar superior de los niños y niñas. Existen centros penitenciarios en México como el Centro de Ejecución de Sanciones Penales, en donde seleccionan a los internos por su buen comportamiento, con ellos se puede trabajar, considerando que reciben un tratamiento integral. Este tipo de metodologías de tratamiento debiesen replicarse en la República Mexicana.

El enfoque en Parentalidad Positiva y la metodología planteada por el programa Triple $\mathrm{P}$, es una herramienta efectiva para el trabajo con padres, madres y cuidadores, ya que cuestiona la paternidad tradicional o estilo parental autoritario, democratizando las responsabilidades, el cuido y la crianza de los hijos e hijas.

Para efectos de este trabajo con padres privados de libertad, fue necesario que se adaptaran las estrategias, su formato y su contenido, adecuándolos a su condición de internamiento. Haciéndolas más vivenciales y socioafectivas, reduciendo el contenido teórico expositivo para mantener su atención. Se hizo necesario detenerse en los estilos parentales y generar espacios para el auto cuestionamiento sobre si el estilo autoritario (predominante en los participantes), vinculado a una paternidad tradicional proveedora, era el mejor estilo considerando la carga emocional que significaba.

En términos generales este trabajo arrojó resultados positivos, en donde se observan cambios a nivel discursivo favorables en cuanto a: concepción de ser padre privado de libertad, a estrategias parentales, a las necesidades de los hijos e hijas, e impacto del internamiento en la familia. También se observaron avances en cuanto a la clarificación de temores y expectativas en relación con su egreso del centro penitenciario. No se observaron cambios significativos a nivel discursivo en cuanto a las estrategias de autocuidado. Con respecto a esto se evidencia que la dinámica parental es atravesada por una cuestión de género.

La socialización masculina está orientada, entre muchos otros aspectos, a no demostrar afecto, a ser el proveedor, a imponer disciplina a través de la violencia, control de la emotividad, desconexión emocional, etc. Estas características fueron identificadas como predominantes en este grupo de padres privados de libertad. En ese sentido, un aspecto pendiente en este trabajo y sumamente indispensable, es la profundización de la perspectiva de género en los estudios con padres privados de libertad. Es necesario que futuras investigaciones integren los aportes de estudios en nuevas masculinidades, paternidades y masculinidad hegemónica.

Aquí se debe manifestar también que uno de los aportes más significativo de este trabajo para los participantes fue el poder generar y abrir espacios de confianza a modo de círculos, en donde los hombres podían adquirir información, pero también se daban las condiciones para que pudieran expresar abiertamente emociones, sentimientos, planes, sueños, problemáticas y sobre todo experiencias relacionadas con sus hijos e hijas. 


\section{BIBLIOGRAFÍA}

Cea, D. M. (2001). Metodología cuantitativa:

Estrategias y técnicas de Investigación

social. Sintesis sociologica.

Departamento Sociología U. Alicante. (s.f.).

Tecnicas de Investigación Social.

https://sites.google.com/site/tecninvestigacionsocial/

temas-y-contenidos/tema-4-las-tecnicas-

estructurales-entrevista-grupo-de-

discusion-observacion-y-biografia/

diseno-de-la-investigacion-cualitativa/tipos-

de-muestreo-cualitativo

Gibbs, G. (2007). Análisis de datos cualitativos en la investigación cualitativa. Morata.

Hernandez, R., Fernández, C., \& Baptista, P. (2010).

Metodología de la investogación. Mc Graw Hill.

Muñiz, M. (2010). Estudios de caso en la investigación cualitativa. https://psico.edu. uy/sites/default/files/cursos/1_estudios-decaso-en-la-investigacion-cualitativa.pdf
Organización de las Naciones Unidas [ONU] (2013). Guia de Introducción a la Preveción de la Reincidencia y la reintegración Social de Delincuentes. Oficina de Naciones Unidas.

Programa en Parentalidad Positiva [Triple P] (2009). Manual del facilitador. Universidad de Queensland.

Subsecretaria de Sistema Penitenciario CDMX. (2020, 31 de julio). Centros de reclusión. https://penitenciario.cdmx.gob.mx/centrosde-reclusion/centro-de-ejecucion-desanciones-penales-varonil-norte

Turner, K., Markie-Dadds, C. Sanders, M. (2013). Manual del facilitador para Triple P Breve. Triple P Internacional. 